\title{
$\begin{array}{ll}\text { Research Square } & \begin{array}{l}\text { Preprints are preliminary reports that have not undergone peer review. } \\ \text { They should not be considered conclusive, used to inform clinical practice, } \\ \text { or referenced by the media as validated information. }\end{array}\end{array}$
}

\section{Metabolically Healthy Versus Unhealthy Obese Phenotypes and Risk of Hypertension Incidence; A Case-Cohort Analysis}

\author{
Behrooz Hamzeh \\ Kermanshah University of Medical Sciences \\ Yahya Pasdar \\ Kermanshah University of Medical Sciences \\ Shima Moradi ( $\sim$ Shima.Moradi@kums.ac.ir) \\ Kermanshah University of Medical Sciences \\ Mitra Darbandi \\ Kermanshah University of Medical Sciences \\ Ebrahim Shakiba \\ Kermanshah University of Medical Sciences \\ Farid Najafi \\ Kermanshah University of Medical Sciences
}

\section{Research Article}

Keywords: metabolically unhealthy obesity, metabolically healthy obesity, hypertension, incidence, PERSIAN

Posted Date: August 9th, 2021

DOI: https://doi.org/10.21203/rs.3.rs-669988/v1

License: (c) (i) This work is licensed under a Creative Commons Attribution 4.0 International License. Read Full License 


\section{Abstract \\ Background}

Although obesity contributes in increasing the risk of hypertension, it is not known the effect of obesity based on metabolic status on the incidence of hypertension. This study was aimed to determine association between obesity phenotypes including metabolically unhealthy obesity (MUO) and metabolically healthy obesity (MHO) and risk of hypertension incidence.

\section{Methods}

We conducted a case-cohort study on 6,747 adults 35-65 aged from Ravansar non- communicable diseases (RaNCD) study. Obesity was defined body mass index $>30 \mathrm{~kg} / \mathrm{m}^{2}$ and metabolically unhealthy was considered at least two metabolic disorders based on the International Diabetes Federation criteria. Obesity phenotypes were categorized four groups including MUO, MHO, metabolically unhealthy non obesity (MUNO), and metabolically healthy non obesity (MHNO). Cox proportional hazards regression models were applied to analyze associations with hypertension incidence.

\section{Results}

The incidence of hypertension was one case per 1000 person-months (393/391162). The MHO (HR: 1.37; 95\% Cl: 1.031.86) and MUO phenotype (HR: 2.44; $95 \% \mathrm{Cl}$ : 1.81-3.29) was linearly associated with higher hypertension risk compared to MHNO. In addition, MUNO phenotype was significantly associated with risk of hypertension incidence (HR: $1.65 ; 95 \%$ Cl: 1.29-2.14).

\section{Conclusions}

Both metabolically healthy and unhealthy obesity was elevated risk of hypertension incidence, however, this increase in metabolically unhealthy phenotypes was higher.

\section{Background}

Hypertension is one of the strongest modifiable risk factors for cardiovascular disease (CVDs) which its prevalence is increasing especially in low- and middle-income countries [1, 2]. In addition to CVDs, hypertension is involved in the pathogenesis of stroke, cerebral hemorrhage, subarachnoid hemorrhage, renal failure, and macrovascular disease [3, 4]. Reports indicate that a quarter of men and a fifth of women have hypertension, and hypertension is responsible for approximately $45 \%$ of deaths from the CVDs $[5,6]$. Results of data from World Health Organization and United Nations Development Program for 182 countries showed that the prevalence of hypertension was 13-41\% [5].

Many factors contribute to hypertension, including sedentary lifestyle, kidney disease, diabetes, obesity, high salt intake and processed foods $[7,8]$. Among these factors, obesity is contributed in the development of CVDs, type 2 diabetes, cancer, inflammatory diseases, and hypertension [9-11]. Evidence suggests that obesity, with its pro-inflammatory effects and oxidative stress, causes insulin resistance, dyslipidemia, and other metabolic disorders in which is considered metabolically unhealthy obesity (MUO) $[12,13]$. Nevertheless, some people with obesity have metabolically healthy status, in which are described metabolically healthy obesity (MHO) phenotype [12]. Additionally metabolically unhealthy non obesity (MUNO) phenotypes are at risk of type 2 diabetes, CVDs, fatty liver, and mortality $[13,14]$. 
Reports indicate that obesity is associated with a risk of developing hypertension. Since a study has not yet examined the types of obesity phenotypes based on the metabolic status of individuals, the present study was conducted with the aim of metabolically healthy versus unhealthy obese phenotypes and risk of hypertension incidence in the Ravansar noncommunicable diseases (RaNCD) cohort study.

\section{Methods}

\section{Study design and setting}

We conducted a case-cohort study nested in the RaNCD cohort The RaNCD study which is a first cohort study on Kurdish population, on aged 35-65 years living in Ravansar city, Kermanshah province, Western- Iran which started in October 2014. The RaNCD cohort study is a component of the PERSIAN (Prospective Epidemiological Research Studies in Iran) mega cohort study that was approved by the Ethics Committees in the Ministry of Health and Medical Education, the Digestive Diseases Research Institute, Tehran University of Medical Sciences, Iran. The details of this study were described in previous studies $[15,16]$. In this study, all recruitment phase participants included, which was surveyed from October 2014 to January 2017 and followed until January 2021 ( $n=4764$ men and 5258 women). The RaNCD cohort study was approved by the Ethics Committee of Kermanshah University of Medical Sciences (No: KUMS.REC.1394.318).

\section{Participants}

Among the RaNCD participants, 3300 of them were not included in the study for the following reasons: 1) participants with CVDs ( $n=1709)$, type 2 diabetes $(n=870)$, hypertension $(n=1579)$, cancer $(n=83)$, and thyroid diseases $(n=763) ; 2)$ pregnant women $(n=138)$; 3 ) energy intake less than $800 \mathrm{Kcal} /$ day or more than $4200 \mathrm{Kcal} /$ day $(n=737)$. After excluding participants with missing data, overall, 6747 participants were included into this study.

\section{Measurements}

This current study was obtained demographic data including age, sex, smoking status, and physical activity, as well as, medical history, medication, anthropometric indices, blood pressure, and biochemical analysis.

\section{Anthropometry}

All participants' height were measured by the automatic stadiometer BSM 370 (Biospace Co., Seoul, Korea) with a precision of $0.1 \mathrm{~cm}$ in standing position without shoes. InBody 770 device (Inbody Co, Seoul, Korea) was applied to measure the weight and body fat mass (BFM) of participants with the least clothing and without shoes. To determine obesity, body mass index (BMI) was calculated by dividing weight in kilogram to square height in meter ${ }^{2}$, after that $\mathrm{BMI}$ more than $30 \mathrm{~kg} / \mathrm{m}^{2}$ as obesity. Waist circumference (WC) was measured using non-stretched and flexible tape in standing position at the level of the iliac crest.

\section{Blood pressure}

In RaNCD cohort study, conventional sphygmomanometry and auscultation of the Korotkoff sounds was used to measure systolic and diastolic blood pressure (SBP and DBP) in sitting position after at least 4-5 minutes of rest. The blood pressure measuring was conducted two times with 10 minutes interval and the mean of them was calculated and reported as the final blood pressure [15].

\section{Biochemical analysis}

25 cc blood samples were collected from all RaNCD participants. The serum and whole blood samples were subdivided and were stored at $-80^{\circ} \mathrm{C}$ the RaNCD cohort laboratory until analysis. Serum fasting blood sugar (FBS) was measured by 
glucose oxidase method. Total cholesterol (TC), high-density lipoproteins (HDL), triglyceride (TG) and low- density lipoproteins (LDL) concentration were measured by enzymatic kits (Pars Azmun, Iran) [15].

\section{Obesity phenotypes}

We defined MUO presence of $\mathrm{BMI}>30 \mathrm{~kg} / \mathrm{m}^{2}$ and at least two metabolic disorder according to the International Diabetes Federation (IDF) statement [17] as follow: $\mathrm{HDL}<40 \mathrm{mg} / \mathrm{dl}$ in men and $<50 \mathrm{mg} / \mathrm{dl}$ in women; increased TG > $150 \mathrm{mg} / \mathrm{dl}$; $\mathrm{SBP}>130 \mathrm{mmHg}$ or DBP $>80 \mathrm{mmHg}$ or antihypertensive medication; and FBS $>100 \mathrm{mg} / \mathrm{dl}$ or medication for diabetes. Also, $\mathrm{MHO}$ was defined $\mathrm{BMI}>30 \mathrm{~kg} / \mathrm{m}^{2}$ and having at most one metabolic disorder mentioned in the previous sentences, as well as, MUNO phenotype was considered presence of $\mathrm{BMI}<30 \mathrm{~kg} / \mathrm{m}^{2}$ and at least two metabolic disorder. In addition, MHNO participants were related to healthy participants without obesity and metabolic disorder.

\section{Outcome measurement hypertension incidence}

The hypertension was defined by codes 110 of the International classification of diseases Tenth Edition (ICD-10), which included SBP/DBP $\geq 140 / 90 \mathrm{mmHg}$ and/or using anti-hypertensive medications in the time interval between baseline (first phase of Ravansar cohort which has been conducted from 2014) and hypertension diagnosis (from 2015 to 2021), which the overall duration of the follow-up was 391162 person-months.

\section{Statistical analysis}

Statistical analysis was performed using Stata, version 14 (Stata Corp, College Station, TX). Mean \pm standard deviation (SD) and frequency percent was used to report baseline characteristics of studied participants. To compare results of baseline characteristics among different obesity phenotypes, one-way analysis of variance (ANOVA) was used for continuous variables, and a Chi-square test was used for categorical variables.

Incidence rate (IR) calculated based on 1000 person/months Cox proportional hazards regression model were used to calculate hazard ratios (HRs) stratified by obesity phenotypes, with hypertension as the event and the time interval between baseline (first phase of RaNCD cohort) and hypertension diagnosis as the time covariate. The models of adjusted for confounding variables including age, sex, physical activity, smoking and energy intake, and reported as HR with $95 \%$ confidence interval $(\mathrm{Cl})$.

\section{Results}

A total of 6,747 participants were analyzed in this study as sub-cohort and 393 incidence cases were also in the subcohort. The incidence rate of hypertension was one cases per 1000 person-months (393/391162, male: 150/188718, female: 243/202443)) during a mean follow-up of 57.74 months (Minimum: 0.27, Maximum: 73.30). In addition, the new case of hypertension was significantly higher in female than male $(6.84 \%$ vs. $4.63 \%, \mathrm{P}<0.001)$. (Table 1$)$ 
Table 1

Baseline characteristics of studied participants

\begin{tabular}{|c|c|c|c|c|c|c|}
\hline Variables & $\begin{array}{l}\text { Total } \\
(n=6747)\end{array}$ & $\begin{array}{l}\text { MHNO } \\
(n=3965)\end{array}$ & $\begin{array}{l}\text { MHO } \\
(n=1036)\end{array}$ & $\begin{array}{l}\text { MUNO } \\
(n=1204)\end{array}$ & $\begin{array}{l}\text { MUO } \\
(n=542)\end{array}$ & \\
\hline Age (year) & $45.77 \pm 7.76^{\star}$ & $45.67 \pm 7.97$ & $44.99 \pm 7.04$ & $46.76 \pm 7.88$ & $45.86 \pm 7.02$ & $\begin{array}{l}<.001 \\
0.001\end{array}$ \\
\hline Weight $(\mathrm{kg})$ & $71.87 \pm 13.42$ & $66.49 \pm 11.02$ & $84.54 \pm 10.83$ & $71.76 \pm 10.11$ & $87.12 \pm 12.25$ & $\begin{array}{l}<.001 \\
0.001\end{array}$ \\
\hline WC (cm) & $96.26 \pm 10.36$ & $92.03 \pm 8.64$ & $106.89 \pm 8.23$ & $95.94 \pm 7.01$ & $107.54 \pm 8.81$ & $\begin{array}{l}<.001 \\
0.001\end{array}$ \\
\hline $\mathrm{BMI}\left(\mathrm{kg} / \mathrm{m}^{2}\right)$ & $27.01 \pm 4.67$ & $24.73 \pm 3.34$ & $33.11 \pm 2.93$ & $26.45 \pm 2.45$ & $33.33 \pm 3.28$ & $\begin{array}{l}<.001 \\
0.001\end{array}$ \\
\hline BFM (kg) & $24.27 \pm 9.41$ & $19.76 \pm 6.61$ & $36.38 \pm 6.78$ & $22.41 \pm 5.25$ & $36.24 \pm 7.61$ & $\begin{array}{l}<.001 \\
0.00\end{array}$ \\
\hline $\mathrm{SBP}(\mathrm{mmHg})$ & $\begin{array}{l}103.55 \pm \\
12.42\end{array}$ & $\begin{array}{l}101.62 \pm \\
11.83\end{array}$ & $\begin{array}{l}104.10 \pm \\
11.31\end{array}$ & $\begin{array}{l}107.16 \pm \\
13.07\end{array}$ & $\begin{array}{l}108.59 \pm \\
13.83\end{array}$ & $\begin{array}{l}<.001 \\
0.00\end{array}$ \\
\hline $\mathrm{DBP}(\mathrm{mmHg})$ & $67.44 \pm 7.82$ & $66.37 \pm 7.36$ & $67.76 \pm 7.51$ & $69.44 \pm 8.28$ & $70.18 \pm 8.95$ & $\begin{array}{l}<.001 \\
0.00\end{array}$ \\
\hline FBS (mg/dl) & $89.92 \pm 9.49$ & $87.91 \pm 8.03$ & $88.96 \pm 8.08$ & $94.36 \pm 11.29$ & $96.58 \pm 11.04$ & $\begin{array}{l}<.001 \\
0.001\end{array}$ \\
\hline $\mathrm{TC}(\mathrm{mg} / \mathrm{dl})$ & $\begin{array}{l}184.01 \pm \\
36.79\end{array}$ & $\begin{array}{l}180.03 \pm \\
37.31\end{array}$ & $\begin{array}{l}186.74 \pm \\
33.72\end{array}$ & $\begin{array}{l}190.40 \pm \\
36.80\end{array}$ & $\begin{array}{l}193.68 \pm \\
34.36\end{array}$ & $\begin{array}{l}<.001 \\
0.001\end{array}$ \\
\hline $\mathrm{TG}(\mathrm{mg} / \mathrm{dl})$ & $\begin{array}{l}130.01 \pm \\
73.75\end{array}$ & $\begin{array}{l}101.75 \pm \\
46.35\end{array}$ & $\begin{array}{l}114.06 \pm \\
47.34\end{array}$ & $\begin{array}{l}205.82 \pm \\
87.54\end{array}$ & $\begin{array}{l}198.37 \pm \\
83.06\end{array}$ & $\begin{array}{l}<.001 \\
0.001\end{array}$ \\
\hline $\mathrm{HDL}(\mathrm{mg} / \mathrm{dl})$ & $46.83 \pm 11.41$ & $49.67 \pm 11.21$ & $49.82 \pm 10.55$ & $38.04 \pm 7.45$ & $39.85 \pm 8.31$ & $\begin{array}{l}<.001 \\
0.00\end{array}$ \\
\hline LDL (mg/dl) & $\begin{array}{l}101.26 \pm \\
24.90\end{array}$ & $98.66 \pm 25.16$ & $\begin{array}{l}102.64 \pm \\
22.76\end{array}$ & $\begin{array}{l}105.89 \pm \\
25.49\end{array}$ & $\begin{array}{l}107.33 \pm \\
22.79\end{array}$ & $\begin{array}{l}<.001 \\
0.00\end{array}$ \\
\hline PA (MET hour/ day( & $41.08 \pm 8.15$ & $41.90 \pm 8.78$ & $39.71 \pm 6.19$ & $40.37 \pm 7.78$ & $39.32 \pm 6.55$ & $\begin{array}{l}<.001 \\
0.0\end{array}$ \\
\hline Current smoking (\%) & 11.9 & 20.7 & 9.4 & 23.1 & 14.1 & $\begin{array}{l}<.001 \\
0.00\end{array}$ \\
\hline $\begin{array}{l}\text { Hypertension } \\
\text { incidence }\end{array}$ & 5.79 & 4.4 & 6.2 & 7.7 & 10.9 & $\begin{array}{l}< \\
0.001\end{array}$ \\
\hline \multicolumn{7}{|l|}{ *Mean \pm SD } \\
\hline \multicolumn{7}{|c|}{ **P-values were obtained one-way ANOVA and Chi square. } \\
\hline \multicolumn{7}{|c|}{$\begin{array}{l}\text { MHNO: metabolically healthy non-obese; MHO: metabolically healthy obese; MUNO: metabolically unhealthy non- } \\
\text { obese; MUO: metabolically unhealthy obese; WC: waist circumference; BMI: body mass index; BFM: body fat mass; } \\
\text { SBP: systolic blood pressure; DBP: diastolic blood pressure; FBS: fasting blood sugar; TC: total cholesterol; TG: } \\
\text { triglyceride; HDL: high density lipoprotein; LDL: low density lipoprotein; PA: physical activity }\end{array}$} \\
\hline
\end{tabular}

The prevalence of MHO, MUNO, and MUO were 15.3, 17.4, and $8.03 \%$; respectively. The mean of physical activity in MHNO was significantly higher than other three obesity phenotypes (MHO, MUNO, and MUO) in both men and women. Table 2 are presented baseline characteristics of studied participants based on the different types of obesity phenotypes. 
Table 2

Baseline characteristics of studied participants based on the different types of obesity phenotypes

\begin{tabular}{|c|c|c|c|c|c|c|c|c|c|c|}
\hline \multirow[t]{3}{*}{ Variables } & \multicolumn{4}{|c|}{ Men $(n=3217)$} & \multirow[t]{3}{*}{$P * *$} & \multicolumn{4}{|c|}{ Women $(n=3530)$} & \multirow[t]{3}{*}{$\mathrm{P} * *$} \\
\hline & MHNO & MHO & MUNO & MUO & & MHNO & MHO & MUNO & MUO & \\
\hline & $\begin{array}{l}(n= \\
2094)\end{array}$ & $\begin{array}{l}(n= \\
246)\end{array}$ & $\begin{array}{l}(n= \\
692)\end{array}$ & $\begin{array}{l}(n= \\
185)\end{array}$ & & $\begin{array}{l}(n= \\
1871)\end{array}$ & $\begin{array}{l}(n= \\
790)\end{array}$ & $\begin{array}{l}(n= \\
512)\end{array}$ & $\begin{array}{l}(n= \\
357)\end{array}$ & \\
\hline Age (year) & $\begin{array}{l}45.95 \\
\pm 7.88^{\star}\end{array}$ & $\begin{array}{l}45.13 \\
\pm 7.09\end{array}$ & $\begin{array}{l}45.96 \\
\pm 7.40\end{array}$ & $\begin{array}{l}44.98 \\
\pm 7.01\end{array}$ & 0.174 & $\begin{array}{l}45.35 \\
\pm 8.06\end{array}$ & $\begin{array}{l}44.94 \\
\pm 7.03\end{array}$ & $\begin{array}{l}47.84 \\
\pm 8.37\end{array}$ & $\begin{array}{l}46.31 \\
\pm 6.99\end{array}$ & $\begin{array}{l}<.001 \\
0.001\end{array}$ \\
\hline Weight (kg) & $\begin{array}{l}71.01 \\
\pm 10.74\end{array}$ & $\begin{array}{l}94.50 \\
\pm 9.53\end{array}$ & $\begin{array}{l}76.69 \\
\pm 8.93\end{array}$ & $\begin{array}{l}95.74 \\
\pm 10.33\end{array}$ & $\begin{array}{l}< \\
0.001\end{array}$ & $\begin{array}{l}61.44 \\
\pm 8.94\end{array}$ & $\begin{array}{l}81.44 \\
\pm 9.22\end{array}$ & $\begin{array}{l}65.11 \\
\pm 7.47\end{array}$ & $\begin{array}{l}82.65 \\
\pm 10.70\end{array}$ & $\begin{array}{l}< \\
0.001\end{array}$ \\
\hline WC (cm) & $\begin{array}{l}92.49 \\
\pm 8.30\end{array}$ & $\begin{array}{l}107.53 \\
\pm 7.70\end{array}$ & $\begin{array}{l}96.14 \\
\pm 6.87\end{array}$ & $\begin{array}{l}106.97 \\
\pm 7.71\end{array}$ & $\begin{array}{l}< \\
0.001\end{array}$ & $\begin{array}{l}91.51 \\
\pm 8.97\end{array}$ & $\begin{array}{l}106.68 \\
\pm 8.39\end{array}$ & $\begin{array}{l}95.66 \\
\pm 7.20\end{array}$ & $\begin{array}{l}107.83 \\
\pm 9.32\end{array}$ & $\begin{array}{l}< \\
0.001\end{array}$ \\
\hline BMI $\left(\mathrm{kg} / \mathrm{m}^{2}\right)$ & $\begin{array}{l}24.36 \\
\pm 3.40\end{array}$ & $\begin{array}{l}32.42 \\
\pm 2.30\end{array}$ & $\begin{array}{l}26.26 \\
\pm 2.52\end{array}$ & $\begin{array}{l}32.37 \\
\pm 2.29\end{array}$ & $\begin{array}{l}< \\
0.001\end{array}$ & $\begin{array}{l}25.15 \\
\pm 3.22\end{array}$ & $\begin{array}{l}33.32 \\
\pm 3.07\end{array}$ & $\begin{array}{l}26.70 \\
\pm 2.34\end{array}$ & $\begin{array}{l}33.82 \\
\pm 3.60\end{array}$ & $\begin{array}{l}<.001 \\
0.001\end{array}$ \\
\hline BFM (kg) & $\begin{array}{l}17.74 \\
\pm 6.24\end{array}$ & $\begin{array}{l}33.77 \\
\pm 6.69\end{array}$ & $\begin{array}{l}20.91 \\
\pm 5.01\end{array}$ & $\begin{array}{l}33.54 \\
\pm 7.17\end{array}$ & $\begin{array}{l}< \\
0.001\end{array}$ & $\begin{array}{l}22.31 \\
\pm 6.18\end{array}$ & $\begin{array}{l}37.32 \\
\pm 6.58\end{array}$ & $\begin{array}{l}24.71 \\
\pm 4.77\end{array}$ & $\begin{array}{l}37.90 \\
\pm 7.41\end{array}$ & $\begin{array}{l}< \\
0.001\end{array}$ \\
\hline $\mathrm{SBP}(\mathrm{mmHg})$ & $\begin{array}{l}103.59 \\
\pm 11.53\end{array}$ & $\begin{array}{l}108.20 \\
\pm 11.10\end{array}$ & $\begin{array}{l}107.77 \\
\pm 12.22\end{array}$ & $\begin{array}{l}110.03 \\
\pm 12.87\end{array}$ & $\begin{array}{l}< \\
0.001\end{array}$ & $\begin{array}{l}99.41 \\
\pm 11.77\end{array}$ & $\begin{array}{l}102.83 \\
\pm 11.08\end{array}$ & $\begin{array}{l}106.33 \\
\pm 14.11\end{array}$ & $\begin{array}{l}107.84 \\
\pm 14.26\end{array}$ & $\begin{array}{l}< \\
0.001\end{array}$ \\
\hline $\begin{array}{l}\text { DBP } \\
(\mathrm{mmHg})\end{array}$ & $\begin{array}{l}67.31 \\
\pm 7.50\end{array}$ & $\begin{array}{l}70.30 \\
\pm 7.65\end{array}$ & $\begin{array}{l}69.98 \\
\pm 8.00\end{array}$ & $\begin{array}{l}71.25 \\
\pm 8.77\end{array}$ & $\begin{array}{l}< \\
0.001\end{array}$ & $\begin{array}{l}65.32 \\
\pm 7.05\end{array}$ & $\begin{array}{l}66.97 \\
\pm 7.29\end{array}$ & $\begin{array}{l}68.72 \\
\pm 8.60\end{array}$ & $\begin{array}{l}69.62 \\
\pm 9.00\end{array}$ & $\begin{array}{l}< \\
0.001\end{array}$ \\
\hline FBS (mg/dl) & $\begin{array}{l}88.32 \\
\pm 8.14\end{array}$ & $\begin{array}{l}89.74 \\
\pm 8.57\end{array}$ & $\begin{array}{l}93.71 \\
\pm 10.90\end{array}$ & $\begin{array}{l}95.53 \\
\pm 9.94\end{array}$ & $\begin{array}{l}< \\
0.001\end{array}$ & $\begin{array}{l}87.45 \\
\pm 7.88\end{array}$ & $\begin{array}{l}88.72 \\
\pm 7.91\end{array}$ & $\begin{array}{l}95.25 \\
\pm 11.74\end{array}$ & $\begin{array}{l}97.12 \\
\pm 11.55\end{array}$ & $\begin{array}{l}< \\
0.001\end{array}$ \\
\hline TC (mg/dl) & $\begin{array}{l}178.53 \\
\pm 36.02\end{array}$ & $\begin{array}{l}187.56 \\
\pm 35.36\end{array}$ & $\begin{array}{l}185.02 \\
\pm 33.58\end{array}$ & $\begin{array}{l}188.54 \\
\pm 30.04\end{array}$ & $\begin{array}{l}< \\
0.001\end{array}$ & $\begin{array}{l}181.72 \\
\pm 38.64\end{array}$ & $\begin{array}{l}186.49 \\
\pm 33.22\end{array}$ & $\begin{array}{l}197.68 \\
\pm 39.64\end{array}$ & $\begin{array}{l}196.34 \\
\pm 36.15\end{array}$ & $\begin{array}{l}< \\
0.001\end{array}$ \\
\hline TG (mg/dl) & $\begin{array}{l}108.41 \\
\pm 51.12\end{array}$ & $\begin{array}{l}134.62 \\
\pm 67.99\end{array}$ & $\begin{array}{l}216.65 \\
\pm 89.80\end{array}$ & $\begin{array}{l}220.14 \\
\pm 86.05\end{array}$ & $\begin{array}{l}< \\
0.001\end{array}$ & $\begin{array}{l}94.28 \\
\pm 39.00\end{array}$ & $\begin{array}{r}107.65 \\
\pm 36.49\end{array}$ & $\begin{array}{l}191.19 \\
\pm 82.24\end{array}$ & $\begin{array}{l}187.09 \\
\pm 79.27\end{array}$ & $\begin{array}{l}< \\
0.001\end{array}$ \\
\hline $\mathrm{HDL}(\mathrm{mg} / \mathrm{dl})$ & $\begin{array}{l}46.37 \\
\pm 9.98\end{array}$ & $\begin{array}{l}44.71 \\
\pm 8.98\end{array}$ & $\begin{array}{l}35.24 \\
\pm 5.92\end{array}$ & $\begin{array}{l}35.29 \\
\pm 5.74\end{array}$ & $\begin{array}{l}< \\
0.001\end{array}$ & $\begin{array}{l}53.39 \\
\pm 11.35\end{array}$ & $\begin{array}{l}51.41 \\
\pm 10.50\end{array}$ & $\begin{array}{l}41.82 \\
\pm 7.64\end{array}$ & $\begin{array}{l}42.22 \\
\pm 8.46\end{array}$ & $\begin{array}{l}<.001 \\
0.001\end{array}$ \\
\hline LDL (mg/dl) & $\begin{array}{l}99.23 \\
\pm 24.50\end{array}$ & $\begin{array}{l}106.41 \\
\pm 23.50\end{array}$ & $\begin{array}{l}103.03 \\
\pm 22.89\end{array}$ & $\begin{array}{l}105.91 \\
\pm 20.73\end{array}$ & $\begin{array}{l}< \\
0.001\end{array}$ & $\begin{array}{l}98.03 \\
\pm 25.87\end{array}$ & $\begin{array}{l}101.46 \\
\pm 22.42\end{array}$ & $\begin{array}{l}109.77 \\
\pm 28.19\end{array}$ & $\begin{array}{l}108.08 \\
\pm 23.78\end{array}$ & $<0.001$ \\
\hline $\begin{array}{l}\text { PA (MET } \\
\text { hour/ day( }\end{array}$ & $\begin{array}{l}43.70 \\
\pm 10.87\end{array}$ & $\begin{array}{l}41.59 \\
\pm 9.87\end{array}$ & $\begin{array}{l}41.31 \\
\pm 9.59\end{array}$ & $\begin{array}{l}40.09 \\
\pm 9.78\end{array}$ & $\begin{array}{l}< \\
0.001\end{array}$ & $\begin{array}{l}39.89 \\
\pm 4.84\end{array}$ & $\begin{array}{l}39.12 \\
\pm 4.30\end{array}$ & $\begin{array}{l}39.10 \\
\pm 3.92\end{array}$ & $\begin{array}{l}38.93 \\
\pm 3.91\end{array}$ & $\begin{array}{l}<.001 \\
0.00\end{array}$ \\
\hline $\begin{array}{l}\text { Current } \\
\text { smoking (\%) }\end{array}$ & 35.9 & 30.7 & 34.8 & 35.3 & 0.462 & 3.7 & 2.8 & 7.1 & 3.1 & 0.001 \\
\hline $\begin{array}{l}\text { Hypertension } \\
\text { incidence }\end{array}$ & 4 & 6.5 & 5.1 & 8.1 & 0.029 & 4.9 & 6.1 & 11.3 & 12.3 & $\begin{array}{l}< \\
0.001\end{array}$ \\
\hline \multicolumn{11}{|l|}{ *Mean \pm SD } \\
\hline \multicolumn{11}{|c|}{ **P-values were obtained one-way ANOVA and Chi square. } \\
\hline \multicolumn{11}{|c|}{$\begin{array}{l}\text { MHNO: metabolically healthy non-obese; MHO: metabolically healthy obese; MUNO: metabolically unhealthy non- } \\
\text { obese; MUO: metabolically unhealthy obese; WC: waist circumference; BMI: body mass index; BFM: body fat mass; } \\
\text { SBP: systolic blood pressure; DBP: diastolic blood pressure; FBS: fasting blood sugar; TC: total cholesterol; TG: } \\
\text { triglyceride; HDL: high density lipoprotein; LDL: low density lipoprotein; PA: physical activity }\end{array}$} \\
\hline
\end{tabular}

The risk increased in MHO phenotype compared to MHNO (HR: 1.41; 95\% Cl: 1.05-1.88) in model I, which remained significant after adjustment for age, sex, physical activity and smoking (HR: 1.37; 95\% Cl: 1.03-1.86). The risk increased 
in MUO phenotype compared to MHNO (HR: 2.44; 95\% Cl: 1.81-3.29) after adjust sex and age, which remained significant after adjustment for age, sex, physical activity and smoking (HR: 2.40; 95\% Cl: 1.77, 3.26). (Table 3)

Table 3

Hazard ratio of incident hypertension according to obesity phenotypes

\begin{tabular}{|c|c|c|c|c|c|}
\hline \multirow[t]{2}{*}{ Obesity phenotypes } & \multirow[t]{2}{*}{$\mathbf{N}$} & \multirow[t]{2}{*}{$\%(N)$ of cases } & \multicolumn{3}{|c|}{ Hazard ratio $(95 \% \mathrm{Cl})$} \\
\hline & & & Model I & Model II & Model III \\
\hline MHNO & 3965 & $4.4(175)$ & Ref. & Ref. & Ref. \\
\hline $\mathrm{MHO}$ & 1036 & $6.2(64)$ & $1.41(1.05,1.88)$ & $1.37(1.02,1.83)$ & $1.37(1.03,1.86)$ \\
\hline MUNO & 1204 & 7.7 (93) & $1.68(1.31,2.16)$ & $1.64(1.27,2.11)$ & $1.65(1.29,2.14)$ \\
\hline MUO & 542 & $10.9(59)$ & $2.44(1.81,3.29)$ & $2.36(1.75,3.20)$ & $2.40(1.77,3.26)$ \\
\hline \multicolumn{6}{|c|}{$\begin{array}{l}\text { Model 1: Adjusted for age and sex; Model 2: Adjusted for age, sex and physical activity; Model 3: Adjusted for age, } \\
\text { sex, physical activity and smoking }\end{array}$} \\
\hline
\end{tabular}

In addition, risk of hypertension significantly increased in MUNO phenotype compared to MHNO in all adjusted models (HR: $1.65 ; 95 \% \mathrm{Cl}: 1.29-2.14)$. The cumulative hazard curves show the incidence of hypertension has increased by approximately $7 \%$ in MUO phenotype over 70 months; and this increase was more than other phenotypes in over time (Fig. 1).

\section{Discussion}

Our results shows both phenotypes obesity $\mathrm{MHO}$ and $\mathrm{MUO}$ increase to develop risk of the hypertension compared to MHNO phenotype. Furthermore, MUNO phenotype was associated with higher risk of hypertension incidence compared to MHNO phenotype. Overall, the MUO phenotype increased risk of hypertension incidence more than other phenotypes in the follow-up study time. The obesity epidemic is growing and increases the risk of chronic non-communicable diseases leading to increased health system costs [18]. Epidemiological studies highlight the persistent link between obesity and hypertension, and the presence of obesity increases the risk of developing hypertension [8, 19]. Since there are different phenotypes of obesity based on the metabolic status, to best our knowledge, we examined the association between obesity phenotypes and risk of hypertension incidence.

The results of Whitehall II cohort study by Hinnouho et al. [20] on 5269 participants indicated that both obesity phenotypes, $\mathrm{MHO}$ and $\mathrm{MUO}$ lead to increased risk of mortality after seventeen years following. Another prospective study by Fingeret et al. [21] after 10.9 years follow up was not seen any difference between $\mathrm{MHO}$ and MUO in hypertension incidence (odds ratio (OR): 1.3, Cl 95\%: 0.8-2.09). Yuan et al. [22] showed that MHO had no association with arterial stiffness developing (OR: 0.99; Cl 95\%: 0.61-1.6), while MUO and MUNO phenotypes lead to significantly progressed arterial stiffness (OR: 4.56; Cl 95\%: 2.60-8) and (OR: 5.05; Cl 95\%: 3.12-8.19), respectively.

In current study we observed that MUO and MUNO increase the risk of hypertension incidence more than MHO. Also, BFM and WC of the participants were higher in all three groups than MHNO phenotype. Obesity, especially the high excess visceral fat distribution is increased inflammatory cytokines and endothelial disorders in which stimulate several mechanisms contribute to hypertension $[18,23]$. High excess adipose tissue increases the production of proinflammatory factors such as leptin, tumor necrosis factor- $a$, interleukin-6, and resistin in which develops various metabolic diseases [24]. High calorie intake and increase in adipocytes stimulate $a$ and $\beta$ adrenergic receptors, thereby 
increasing the activity of the sympathetic nervous system [25]. Obesity activates the renin-angiotensin nervous system and the sympathetic nervous system, which leads to increased sodium reabsorption and arterial blood pressure [26, 27]. On the other hand, increasing adipose tissue leads to decreased adiponectin production and increased insulin resistance $[28,29]$. Therefore, chronic hyperinsulinemia in obese people causes vascular vasoconstrictor and also increases urinary sodium reabsorption and is involved in the pathogenesis of hypertension [30]. Also, increased circulating leptin levels in response to increased adipose tissue lead to impaired nitric oxide synthesis and ultimately vascular endothelial dysfunction [18]. Therefore, obesity increases the production of adipose tissue, causing the production of proinflammatory cytokines, which play an important role in the pathogenesis of hypertension by disrupting the metabolic status.

\section{Strength And Limits}

The present prospective study follows for the first time the Kurdish population and examines the types of obesity based on metabolic status and risk of hypertension incidence. In this study, we also applied appropriate exclusion criteria, such as people who did not have normal calorie intake. However, this study had its limitations. First, the follow-up period seems to have been short. Second, the hypertension incidence was small for the study groups, and we could not assess the relationship between hypertension incidence and obesity phenotypes based on the sex, although it was adjusted for sex.

\section{Conclusion}

In conclusion, present study stated that both of $\mathrm{MHO}$ and $\mathrm{MUO}$ phenotypes lead to rise hypertension incidence compared to MHNO phenotype, as well as, MUNO phenotype can increase hypertension incidence. However, MUO and MUNO phenotypes increase the risk of hypertension incidence more than $\mathrm{MHO}$ compared to MHNO phenotype. For protecting from hypertension maintaining normal weight and controlling central obesity as well as visceral fat is highly recommended.

\section{Declarations}

\section{Acknowledgments}

RaNCD is part of PERSIAN national cohort and we would like to thank Professor Reza Malekzadeh, Ex-Deputy of Research and Technology at the Ministry of Health and Medical Education of Iran and Director of the PERSIAN cohort, and also Dr. Hossein Poustchi Executive Director of PERSIAN cohort for all their supports during design and running of RaNCD. We gratefully acknowledge our RaNCD field workers and site staffs and participants of the RaNCD cohort for their important contributions.

Funding: This study was supported by Ministry of Health and Medical Education of Iran and Kermanshah University of Medical Science (Grant No: 92472).

\section{Compliance with ethical standards}

Ethics approval and consent to participate: All procedures performed in studies involving human participants were in accordance with the ethical standards of the institutional and/or national research committee and with the $1964 \mathrm{Helsinki}$ declaration and its later amendments or comparable ethical standards. This study was approved by the Ethics Committee of Kermanshah University of Medical Sciences (ethics approval number: KUMS.REC.1394.318).

Informed consent: Written informed consent was obtained from each studied subject after explaining the purpose of the study. The right of subjects to withdraw from the study at any time and subject's information is reserved and will not be 
published.

\section{Consent for publication: Not applicable}

Availability of data and materials: Data will be available upon request from the corresponding author.

Competing interests: All authors have no conflict of interest.

Authors' contributions: F, Njafi, B, Hamzeh, S, Moradi and Y, Pasdar equally contributed to the conception and design of the research; F, Njafi, B, Hamzeh, E, Shakiba and Y, Pasdar contributed to data collection; S, Moradi, Y, Pasdar and M, Darbandi contributed to the acquisition and analysis of the data; S, Moradi, Y, Pasdar and M, Darbandi contributed to the interpretation of the data; and S, Moradi, Y, Pasdar and M, Darbandi contributed to draft the manuscript. All authors are in agreement with the manuscript and declare that the content has not been published elsewhere.

\section{References}

1. Mills KT, Stefanescu A, He J. The global epidemiology of hypertension. Nature Rev Nephrol. 2020;16(4):223-37.

2. Fisher ND, Curfman G. Hypertension-a public health challenge of global proportions. Jama. 2018;320(17):1757-9.

3. Umemura S, Arima H, Arima S, Asayama K, Dohi Y, Hirooka Y, et al. The Japanese Society of Hypertension guidelines for the management of hypertension (JSH 2019). Hypertension Res. 2019;42(9):1235-481.

4. Kjeldsen SE. Hypertension and cardiovascular risk: general aspects. Pharmacol Res. 2018;129:95-9.

5. Zeng Z, Chen J, Xiao C, Chen W. A Global View on Prevalence of Hypertension and Human Develop Index. Ann Global Health. 2020;86(1).

6. Egan BM, Kjeldsen SE, Grassi G, Esler M, Mancia G. The global burden of hypertension exceeds 1.4 billion people: should a systolic blood pressure target below 130 become the universal standard? J Hypertension. 2019;37(6):1148-53.

7. Eze II, Mbachu CO, Azuogu BN, Ossai E, Unah Al, Akamike IC, et al. Effect of on-site behavioural modification intervention on lifestyle risk factors of hypertension among adult market traders in Abakaliki, Nigeria. Inter J Health Promotion Edu. 2021;59(1):35-49.

8. Ostchega Y, Zhang G, Hughes JP, Nwankwo T. Factors associated with hypertension control in US adults using 2017 ACC/AHA guidelines: National Health and Nutrition Examination Survey 1999-2016. Am J Hypertension. 2018;31(8):886-94.

9. Avgerinos KI, Spyrou N, Mantzoros CS, Dalamaga M. Obesity and cancer risk: Emerging biological mechanisms and perspectives. Metabolism. 2019;92:121-35.

10. Koliaki C, Liatis S, Kokkinos A. Obesity and cardiovascular disease: revisiting an old relationship. Metabolism. 2019;92:98-107.

11. Trends in obesity and diabetes across Africa from 1980 to 2014: an analysis of pooled population-based studies. Inter J Epidemiol. 2017;46(5):1421-32.

12. Có'toi AF, Pârvu AE, Andreicuț $A D$, Mironiuc A, Cró'ciun A, Có'toi $C$, et al. Metabolically healthy versus unhealthy morbidly obese: chronic inflammation, nitro-oxidative stress, and insulin resistance. Nutrients. 2018;10(9):1199.

13. Kim Y, Chang Y, Cho YK, Ahn J, Shin H, Ryu S. Metabolically healthy versus unhealthy obesity and risk of fibrosis progression in non-alcoholic fatty liver disease. Liver Inter. 2019;39(10):1884-94.

14. Hsu ARC, Ames SL, Xie B, Peterson DV, Garcia L, Going SB, et al. Incidence of diabetes according to metabolically healthy or unhealthy normal weight or overweight/obesity in postmenopausal women: the Women's Health Initiative. Menopause. 2020;27(6):640-7. 
15. Pasdar Y, Najafi F, Moradinazar M, Shakiba E, Karim H, Hamzeh B, et al. Cohort profile: Ravansar Non-Communicable Disease cohort study: the first cohort study in a Kurdish population. International journal of epidemiology. 2019;https://doi.org/10.1093/ije/dyy296.

16. Poustchi H, Eghtesad S, Kamangar F, Etemadi A, Keshtkar A-A, Hekmatdoost A, et al. Prospective epidemiological research studies in Iran (the PERSIAN Cohort Study): rationale, objectives, and design. American journal of epidemiology. 2017;187(4):647-55.

17. Alberti KGM, Zimmet P, Shaw J. The metabolic syndrome-a new worldwide definition. The Lancet. 2005;366(9491):1059-62.

18. Leggio M, Lombardi M, Caldarone E, Severi P, D'emidio S, Armeni M, et al. The relationship between obesity and hypertension: an updated comprehensive overview on vicious twins. Hypertension Res. 2017;40(12):947-63.

19. Ruilope LM, Nunes Filho A, Nadruz Jr W, Rosales FR, Verdejo-Paris J. Obesity and hypertension in Latin America: Current perspectives. Hipertension y riesgo vascular. 2018;35(2):70-6.

20. Hinnouho G-M, Czernichow S, Dugravot A, Batty GD, Kivimaki M, Singh-Manoux A. Metabolically healthy obesity and risk of mortality: does the definition of metabolic health matter? Diabetes care. 2013;36(8):2294-300.

21. Fingeret $M$, Marques-Vidal P, Vollenweider P. Incidence of type 2 diabetes, hypertension, and dyslipidemia in metabolically healthy obese and non-obese. Nutrition, Metabolism Cardiovasc Dis. 2018;28(10):1036-44.

22. Yuan Y, Mu J-J, Chu C, Zheng W-L, Wang Y, Hu J-W, et al. Effect of metabolically healthy obesity on the development of arterial stiffness: a prospective cohort study. Nutrition Metabolism. 2020;17(1):1-10.

23. Seravalle G, Grassi G. Obesity and hypertension. Pharmacol Res. 2017;122:1-7.

24. Jiang $P$, Ma D, Wang X, Wang Y, Bi Y, Yang J, et al. Astragaloside IV prevents obesity-associated hypertension by improving pro-inflammatory reaction and leptin resistance. Molecule Cell. 2018;41(3):244.

25. Lambert EA, Straznicky NE, Dixon JB, Lambert GW. Should the sympathetic nervous system be a target to improve cardiometabolic risk in obesity? Am J Physiol Heart Circ Physiol. 2015;309(2):H244-H58.

26. Aronow WS. Association of obesity with hypertension. Ann Translational Med. 2017;5(17).

27. Cwynar M, Gąsowski J, Gryglewska B, Głuszewska A, Kwater A, Królczyk J, et al. Insulin resistance and renal sodium handling influence arterial stiffness in hypertensive patients with prevailing sodium intake. Am J Hypertension. 2019;32(9):848-57.

28. Ohashi K, Kihara S, Ouchi N, Kumada M, Fujita K, Hiuge A, et al. Adiponectin replenishment ameliorates obesityrelated hypertension. Hypertension. 2006;47(6):1108-16.

29. De Boer MP, Meijer RI, Wijnstok NJ, Jonk AM, Houben AJ, Stehouwer CD, et al. Microvascular dysfunction: a potential mechanism in the pathogenesis of obesity-associated insulin resistance and hypertension. Microcirculation. 2012;19(1):5-18.

30. Soleimani M. Insulin resistance and hypertension: new insights. Kidney Inter 2015;87(3):497-9.

\section{Figures}




\section{Kaplan- Meier failure functions}

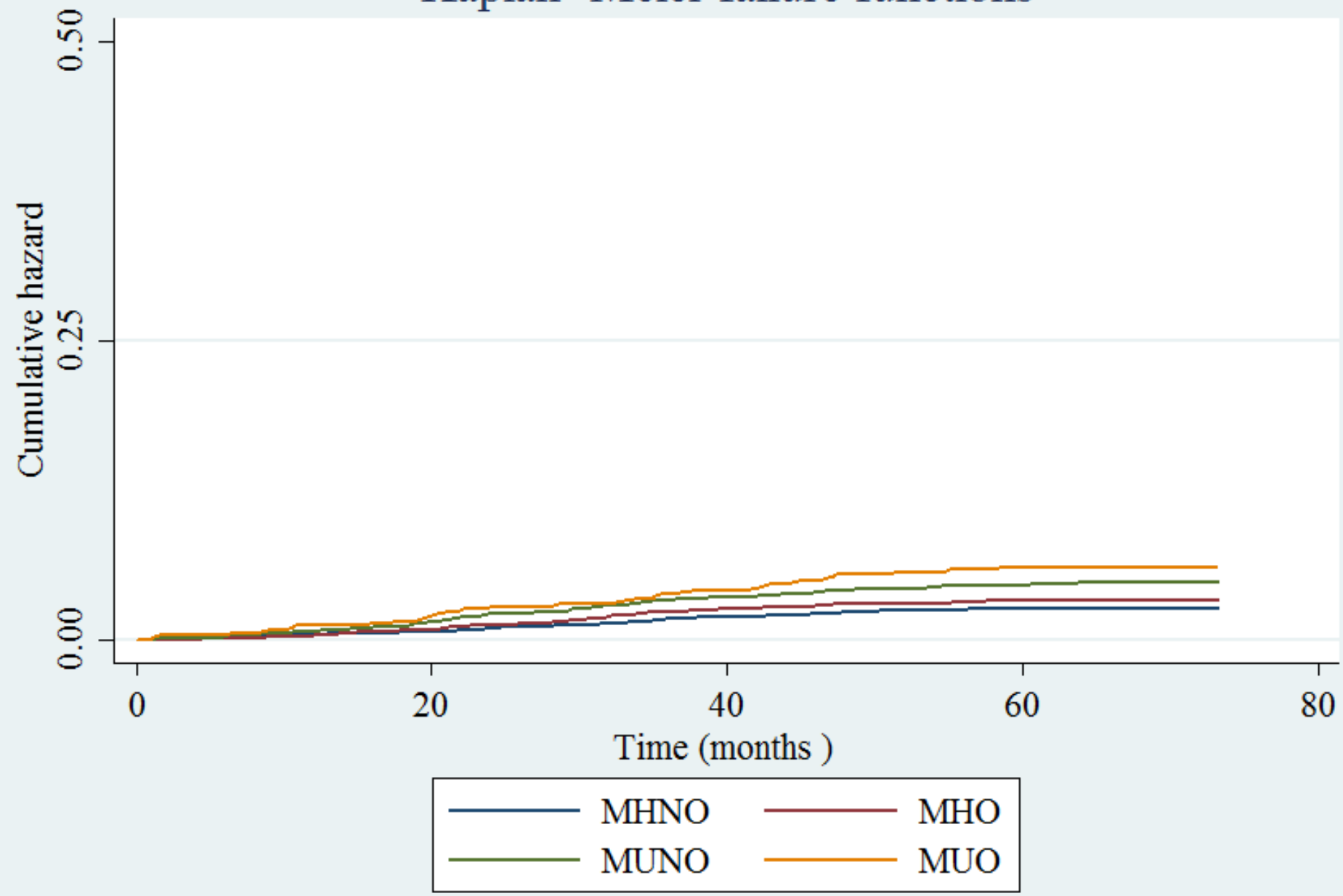

Figure 1

Cumulative hazard curves for the incidence of hypertension in over time according to obesity phenotypes 